\title{
Split-Extended LDPC codes for coded cooperation
}

\author{
Valentin Savin, CEA-LETI, MINATEC, Grenoble, France, valentin.savin@cea.fr
}

\begin{abstract}
We propose a new code design that aims to distribute an LDPC code over a relay channel. It is based on a splitand-extend approach, which allows the relay to split the set of bits connected to some parity-check of the LDPC code into two or several subsets. Subsequently, the sums of bits within each subset are used in a repeat-accumulate manner in order to generate extra bits sent from the relay toward the destination. We show that the proposed design yields LDPC codes with enhanced correction capacity and can be advantageously applied to existing codes, which allows for addressing cooperation issues for evolving standards. Finally, we derive density evolution equations for the proposed design, and we show that Split-Extended LDPC codes can approach very closely the capacity of the Gaussian relay channel.
\end{abstract}

\section{INTRODUCTION}

By exploiting the broadcast nature and the inherent spatial diversity of wireless communications, Sendonaris et al. introduced the concept of cooperative diversity [1], [2] over wireless relay channels and their multi-terminal extensions. A relay channel is a three terminal network consisting of a source, a relay, and a destination. The source broadcasts a message to both relay and destination, while the relay forwards the message to the destination. Subsequently, many authors proposed cooperation protocols for the relay channel, which can be classified into two major categories, namely the amplify-and-forward (AF) and the decode-and-forward (DF) [3]. In AF protocols, the relay simply amplifies the received signal and forwards it to the destination. The DF protocol allows the relay to decode the received signal, re-encode it, and forward it to the destination. The forwarded message can either be identical to, or part of the initial transmission (repetition coding), or it can be obtained by using a dedicated coding scheme at the relay (distributed coding). In the first case the destination combines received signals from both source and relay, which results in an improved signal-to-noise ratio (SNR) on the received transmission. Besides, the same code is used for encoding at the source and decoding at the destination. In the second case, the destination gains knowledge of extra information, but it needs a dedicated decoding scheme, able to jointly decode received signals from both source and relay.

One of the most known examples of distributed coding is the one of a distributed turbo-code [4]: the source broadcasts a recursive convolutional code (RCC) to both relay and destination. After decoding, the relay interleaves and re-encodes the message using the same RCC, prior to forwarding it to the destination. Because the destination receives both codes in parallel, it can jointly decode received signals from source and relay by using a parallel-concatenated turbo-code.

This work was partially carried out in the scope of Celtic CP5-026 (WINNER+) project.
Low-Density Parity-Check (LDPC) codes play a prominent role in the family of error-correcting codes. They feature low complexity decoding and can be optimized for a broad class of channels, with performance approaching the theoretical Shannon limit [5]. Although they lend themselves less easily to distributed schemes, several approaches have been already proposed in the literature [6], [7], [8], [9], [10], [11], [12], [13]. Some of these approaches are somehow based either on serial or parallel code concatenation 1 , or on punctured (ratecompatible) LDPC codes. From the code design point of view, the serial or parallel concatenation of LDPC codes has intrinsic limitations, mainly because parity-check matrices used for decoding at the relay and at the destination are included one in the other, resulting in inappropriate matrix topologies (density on non-zero entries, column and row weight distributions, cycles, etc.). Punctured codes also present some weaknesses in the context of coded cooperation. In such a cooperation scheme, punctured bits, which are not broadcasted by the source, are transmitted from the relay toward the destination. Hence, the punctured code has to be robust, such as to allow successful decoding at the relay: in practice punctured bits are those bits which are the easiest to retrieve by the iterative decoding process. On the other hand, bits transmitted from the relay to the destination encounter better channel conditions: advantageously, these bits should be those which are the most difficult to retrieve by the iterative decoding process. The contradiction between these two requirements leads to an imbalance in the design of the puncturing pattern.

In this paper we propose a new code design method that aims to create incremental redundancy for LDPC codes, while avoiding both code concatenation and code puncturing. It is based on a split-and-extend approach, which can be seen as the "coding analogous of the divide-and-conquer concept". After decoding the received signal, the relay computes extra parity bits by splitting parity-checks of the initial code. Hence, each extra parity bit is the sum of some subset of bits participating in the same parity-check of the initial code. Then the relay transmits these new parity bits towards the destination. The whole process amounts to create a new matrix, whose rows correspond to parity-checks involving both old and new parity bits. These parity-checks are therefore distributed over the relay channel, in the sense that part of checked bits are received on the source-to-destination link, and another part are received on the relay-to-destination link. Consequently, this new matrix can be used at the destination to jointly decode the received signals from both the source and relay.

\footnotetext{
${ }^{1}$ Meaning that the graph of the LDPC code broadcasted from the source is a subgraph of the destination decoding graph.
} 
The paper is organized as follows. The proposed code design is introduced in Section [II In Section [II] we show that the proposed design can be advantageously applied to to existing codes, which allows for backward compatibility while addressing cooperation issues for evolving standards. In Section IV we propose a "coding-perspective" analysis of cooperative systems. We introduce threshold and capacity functions, and we derive density evolution equations for SplitExtended (SE) LDPC codes over the Gaussian relay channel.

Numerical results are shown in Section $\mathrm{V}$, and Section VI concludes this paper.

\section{SPlit-EXTEnded LDPC CODES}

\section{A. Basic idea}

The basic idea of the split-extend design can be resumed as follows. Let $H_{1}$ be the parity-check matrix of the LDPC code broadcasted by the source to both relay and destination. Hence, broadcasted bits satisfy parity-check equations corresponding to the rows of $H_{1}$. After decoding the received signal, the relay computes extra parity bits by splitting these parity-checks, as illustrated at Figure 1. The parity-check in the middle corresponds to a row of $H_{1}$. In the left example, a new parity bit $e_{1}$ is created by spitting the original parity-check into two sub-checks. Precisely, this means that the set of bits connected to the check-node is partitioned into two subsets, and the parity bit $e_{1}$ is generated as the sum of the bits of either one of the two subsets. In the right example, two new parity bits $e_{1}$ and $e_{2}$ are created by spitting the original parity-check into three parity-checks. Precisely, the set of bits connected to the checknode is partitioned into three subsets, and $e_{1}$ is generated as the sum of the bits in the first subset. Subsequently, $e_{2}$ can be generated either as the sum of $e_{1}$ and the bits in the second subset, or as the sum of the bits in the third subset. The total number of extra parity bits depends on the number of rows of $H_{1}$ and the number of extra bits generated for each row of $H_{1}$ (which may vary from one row to another). The sequence of all the extra parity bits, denoted by $E=\left(e_{1}, e_{2}, \ldots\right)$, is then transmitted from the relay to the destination. The matrix $H$ obtained by the split-extension of $H_{1}$ (i.e. the incidence matrix of the split-extended graph) verifies $H \cdot(X, E)^{t}=0$, where $X$ denotes the codeword broadcasted by the source. Therefore, $H$ can be used at the destination in order to jointly decode the received signals from both source and relay.

A more general example of split-extension is illustrated at Figure 2 The original check-node is split into several subchecks, and extended bits are generated in a repeat-accumulate manner. Such a split-extension will be referred to latter in the paper (Section IV) as repeat-accumulate split-extension.

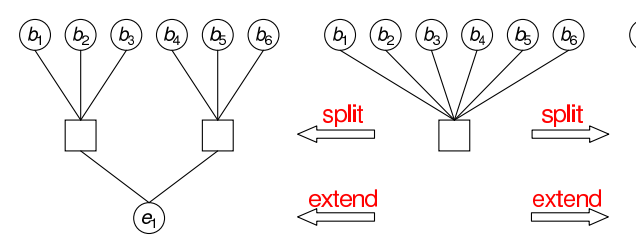

Fig. 1. Split-Extension examples

\section{B. The general case}

The following notation will be used throughout this section:

- For any positive integer $N,[1: N]=\{1, \ldots, N\}$ denotes the set of integers between 1 and $N$, inclusive.

- For any subset $S \subset[1: N],[1: N] \backslash S$ denotes the set of integers between 1 and $N$ that are not in $S$.

- For any length- $N$ vector $V=\left(v_{1}, \ldots, v_{N}\right)$ and any subset $S=\left\{i_{1}, \ldots, i_{k}\right\} \subset[1: N],\left.V\right|_{S}$ denotes the length- $k$ vector defined by the coordinates of $V$ which are in $S$, that is $\left.V\right|_{S}=\left(v_{i_{1}}, \ldots, v_{i_{k}}\right)$

- For any binary matrix $H$ of size $M \times N, \mathcal{R}(H) \subset\{0,1\}^{N}$ denotes the set of the $M$ row-vectors of $H$. A partition of $\mathcal{R}(H)$ is a set of nonempty subsets $\mathcal{P}_{1}, \ldots, \mathcal{P}_{M_{1}} \subseteq \mathcal{R}(H)$ such that every row of $H$ is in exactly one of these subsets.

- For any binary vectors $V_{1}, V_{2} \in\{0,1\}^{N}, V_{1} \oplus V_{2}$ denotes their componentwise sum modulo 2.

[Definition] Let $H_{1}$ and $H$ be two parity-check matrices of size $M_{1} \times N_{1}$ and $M \times N$, respectively, with $N_{1} \leq N$. We say that the matrix $H$ is obtained by split-extending the matrix $H_{1}$ if there exist a partition of the $M$ rows of $H$ in $M_{1}$ disjoint subsets $\mathcal{P}_{1}, \ldots, \mathcal{P}_{M_{1}} \subseteq \mathcal{R}(H)$, and a subset $S \subset[1: N]$ with cardinality $N_{1}$, such that for any $m \in\left[1: M_{1}\right]$ :

$$
\left.\bigoplus_{L \in \mathcal{P}_{m}} L\right|_{S}=R_{m} \text { and }\left.\bigoplus_{L \in \mathcal{P}_{m}} L\right|_{[1: N] \backslash S}=0,
$$

where $R_{1}, \ldots, R_{M_{1}}$ denote the $M_{1}$ rows of $H_{1}$. In this case, the set $E=[1: N] \backslash S$ is called set of extended bit-nodes. The split-extension is called non-singular if the columns of $H$ corresponding to $E$ are linearly independent.

Now, consider two parity-check matrices $H_{1}$ and $H$, such that $H$ is a non-singular split-extension of $H_{1}$. Then:

- If $X$ is a codeword2 of $H$, then $\left.X\right|_{S}$ is a codeword of $H_{1}$

- For any codeword $X_{1}$ of $H_{1}$, there exists a unique codeword $X$ of $H$, such that $\left.X\right|_{S}=X_{1}$

Matrices $\left(H_{1}, H\right)$ can be used within a cooperative transmission system as follows:

- The source encodes the packet of information bits, generating a codeword $X_{1}$ of $H_{1}$. It broadcasts $X_{1}$ to both relay and destination.

- The relay decodes the received signal, correcting the transmission errors on $X_{1}$. It generates a codeword $X$ of $H$, such that $\left.X\right|_{S}=X_{1}$, and sends the set of extended bits $\left.X\right|_{E}$ towards the destination.

- Thus, the destination receives noisy versions of $\left.X\right|_{S}=X_{1}$ and $\left.X\right|_{E}$ (from both the source and the relay), which can be decoded using the matrix $H$.

${ }^{2}$ By abusing language, we say that $X$ is a codeword of $H$, if $H X^{t}=0$
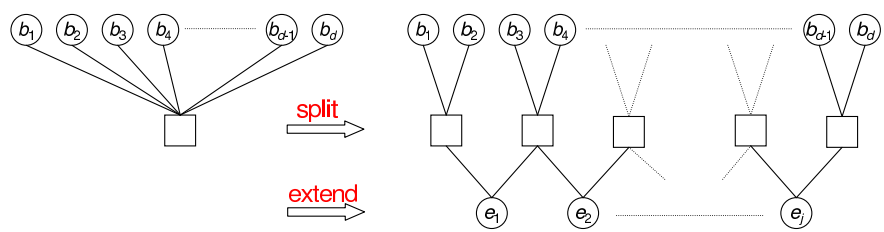

Fig. 2. Repeat-Accumulate Split-Extension 


\section{SPLIT-EXTEND DESIGN FOR BACKWARD COMPATIBILITY}

This section is independent of the following sections, though, it highlights an interesting property of the proposed design: it can be advantageously applied to existing codes, which allows for addressing cooperation issues for evolving standards, while maintaining backward compatibility with a reduced impact on user equipment. To illustrate this, the LDPC codes from the IEEE.802.16e (WiMAX) standard [14] with coding rates $1 / 2$ and $2 / 3$ have been split-extended, such that the number of generated extended bits be equal to the number of information bits. Thus, for coding rate $1 / 2$, each row of the parity-check matrix has been split into two rows; while for rate $2 / 3$, each row of the parity-check matrix has been split into three rows (see Figure 1). Splitting has been performed by a dedicated algorithm that search for short cycles in the parity check matrix, then splits rows such that to break as many short cycles as possible. Base matrix of the Quasi-Cyclic (QC) LDPC WiMAX code with rate 1/2 and the corresponding split-extended matrix are shown at Figure 3 ( -1 's entries of the base matrix are represented by a dash sign).

Clearly, split-extended matrices can be used to address cooperation issues for uplink transmissions, in a completely transparent way for the user: the user encodes the transmitted signal by using the original parity check matrix; the relay decodes the signal, then computes and sends the sequence of extended parity bits to the base station, which will use the split-extended matrix in order to decode the received signals from both user and relay. Hence, split-extended matrix is only needed at the relay and the base station. For downlink transmissions, if the user terminal is equipped with split-extended matrices, the situation is symmetric. Otherwise, the relay can only repeat the sequence of information bits, which, however, provides the user with an energy gain on the information sequence.

Simulation results over the AWGN relay channel, with QPSK modulation, are shown at Figures 4 and 5. The source broadcasts either the WiMAX code with rate $1 / 2$ (Fig. 4) or the WiMAX code with rate $2 / 3$ (Fig. 5), and the SNR on the source-to-relay link is fixed to 2.5 and $4.5 \mathrm{~dB}$, respectively. Both figures compare the performance of two cooperation scenarios: the relay generates and transmits extended bits in the first scenario, while in the second, it forwards the (error-corrected) sequence of information bits. Plotted curves represent SNRs required on source-to-destination and relay-todestination links, such that to obtain a target frame error rate either of $10^{-2}$ (dotted curves) or of $10^{-4}$ (solid curves). The Self-Corrected Min-Sum algorithm [15] is used for decoding at both relay and destination. The gap between the dotted and solid curves is determined by the slope of the frame error rate curves in the waterfall region. However, for the second scenario (repetition of the information sequence) the increased gap between the two curves for small $\mathrm{SNR}_{\mathrm{SD}}$ values is also justified by a frame error rate error floor above $10^{-4}$. Note also that only SNR pairs with $\mathrm{SNR}_{\mathrm{RD}}>\mathrm{SNR}_{\mathrm{SD}}$ are likely to

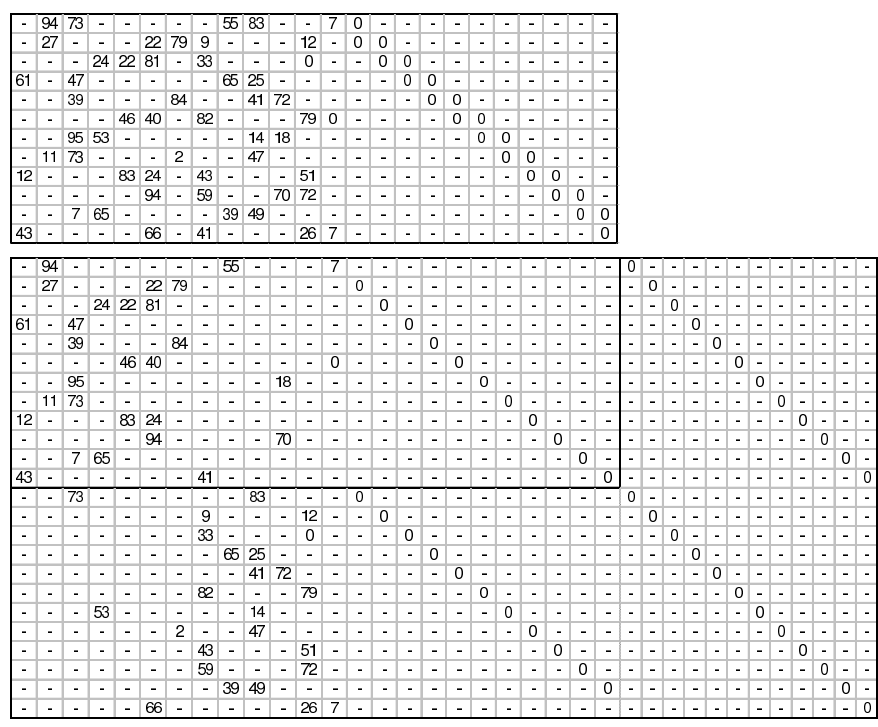

Fig. 3. Base matrix of the QC-LDPC WiMAX code with rate 1/2 (top), and corresponding split-extended base matrix (bottom)

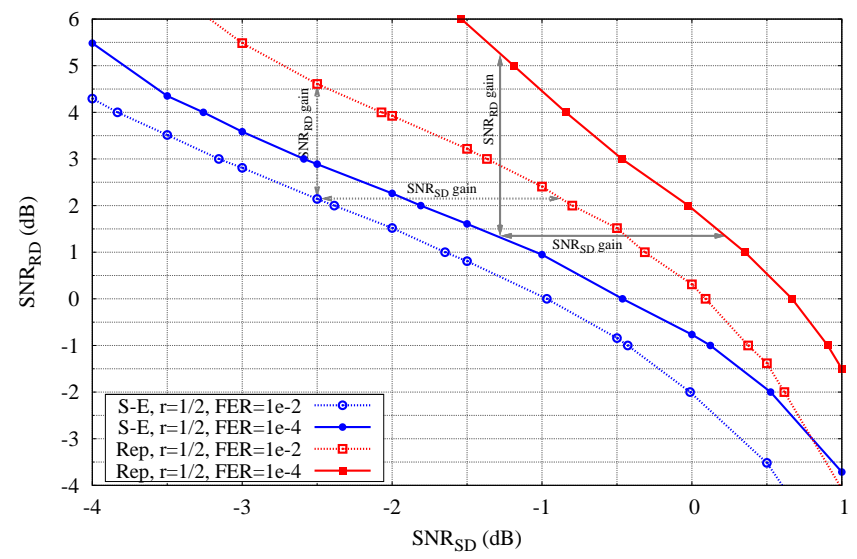

Fig. 4. Split-Extend vs. Repetition coding for WiMAX code with rate $1 / 2$

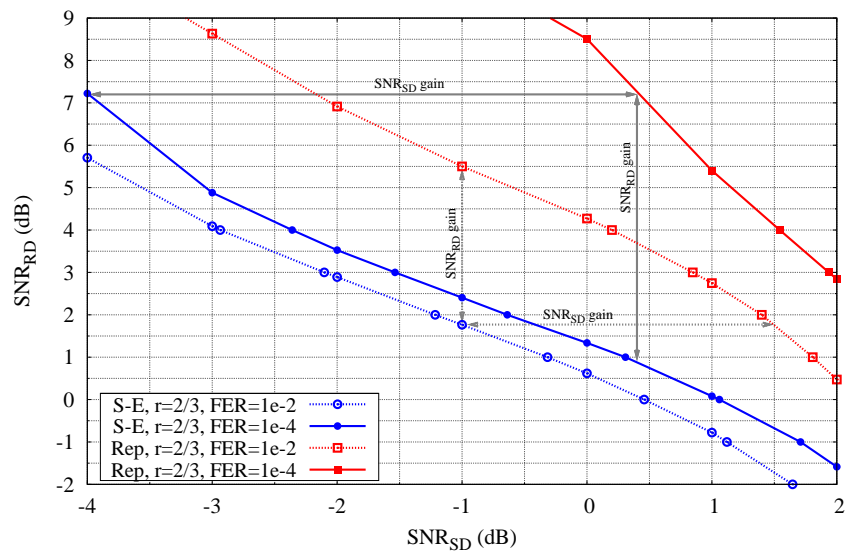

Fig. 5. Split-Extend vs. Repetition coding for WiMAX code with rate $2 / 3$

be encountered in practice. The SNR gain between the two scenarios can be measured either as the horizontal distance (for the source-to-destination link) or the vertical distance (for the relay-to-destination link) between corresponding curves. It can be observed that split-extended codes achieve a significant SNR gain, in order of several dBs, over the repetition scenario. 


\section{ASYMPTOTIC ANALYSIS OF SE-LDPC CODES}

We denote by $\mathcal{E}(\lambda, \rho)$ the ensemble of LDPC codes with edge-perspective degree distribution polynomials $\lambda$ and $\rho$ [16]. It is well known that when the code length tends to infinity, (almost) all the codes of the family behave alike, and they exhibit a threshold phenomenon, separating the region where reliable transmission is possible from that where it is not [16].

Consider some channel model depending on a parameter $\sigma$, such that the channel conditions worsen when $\sigma$ increases (for instance, the noise variance for the AWGN channel, or the error probability for the BSC channel). The threshold of the ensemble $\mathcal{E}(\lambda, \rho)$ is defined as the supremum value of $\sigma$ (worst channel condition) that allows transmission with an arbitrary small error probability, assuming that the transmitted data is encoded with an arbitrary-length code of $\mathcal{E}(\lambda, \rho)$.

The above threshold phenomenon can be extended to the relay channel, but the following must be taken into account:

- the channel is modeled by three parameters $\sigma_{\mathrm{SR}}, \sigma_{\mathrm{SD}}$, and $\sigma_{\mathrm{RD}}$, corresponding, with obvious notation, to the three links between source, relay, and destination.

- the ensemble of SE-LDPC codes depends not only on $\lambda$ and $\rho$, but also on the splitting distribution.

[Channel assumptions] Since we are strictly interested on code analysis, the following assumptions will be made:

- when the relay fails to decode the received signal from the source, it does not transmit any information to the destination,

- the relay channel is degraded, in the sense that the above parameters must satisfy $\sigma_{\mathrm{SR}}<\sigma_{\mathrm{SD}}$ and $\sigma_{\mathrm{RD}}<\sigma_{\mathrm{SD}}$.

[Distributed code] A linear distributed code of dimension $K$ is a vector subspace $\mathcal{C} \subset \mathbb{F}_{2}^{N_{1}} \times \mathbb{F}_{2}^{N_{2}}$, such that $\mathcal{C}$ and its projection on $\mathbb{F}_{2}^{N_{1}}$ are both of dimension $K$. The distributed rate of $\mathcal{C}$ is by definition $\left(r_{1}, r_{2}\right)=\left(\frac{K}{N_{1}}, \frac{K}{N_{2}}\right)$. Hence, $r_{1} \leq 1$, but $r_{2}$ can be greater than 1 . The overall coding rate is defined as $r=\frac{K}{N_{1}+N_{2}}=\frac{r_{1} r_{2}}{r_{1}+r_{2}}$. The idea behind is that the first $N_{1}$ bits of a codeword $c \in \mathcal{C}$ are broadcasted from the source to both relay and destination and, in case that the relay manages to decode the received signa 3 , it transmits the last $N_{2}$ bits toward the destination.

[SE-LDPC ensembles] Let $H_{1}$ be the parity-check matrix of the LDPC code broadcasted by the source, and let $\theta \geq 2$. For each parity-check of $H_{1}$, assume that:

- the set of bits connected to the parity-check is partitioned into $\theta$ subsets of (almost) regular size; that is, each subset contains either $\left\lfloor\frac{d}{\theta}\right\rfloor$ or $\left\lceil\frac{d}{\theta}\right\rceil$ bits participating in the paritycheck, where $d$ denotes the parity-check degree,

- extended bits are generated in a repeat-accumulate manner (Figure 2).

The resulting distributed SE-LDPC code will be referred to as having splitting degree $\theta$. We denote by $\mathcal{E}_{\theta}(\lambda, \rho)$ the ensemble of repeat-accumulate SE-LDPC with splitting degree $\theta$, obtained by split-extending LDPC codes with edge-perspective degree distribution polynomials $\lambda$ and $\rho$.

\footnotetext{
${ }^{3}$ Thus, this definition is dependent on the above channel assumptions.
}

\section{A. SE-LDPC ensemble thresholds}

Given an ensemble of SE-LDPC codes, our intention is to separate the region of parameters $\left(\sigma_{\mathrm{SR}}, \sigma_{\mathrm{SD}}, \sigma_{\mathrm{RD}}\right)$ where reliable transmission is possible from that where it is not.

Let $\sigma_{1}^{*}(\lambda, \rho)$ be the threshold of the $\mathcal{E}(\lambda, \rho)$ ensemble. If $\sigma_{\mathrm{SR}}>\sigma_{1}^{*}(\lambda, \rho)$, the error decoding probability at the relay is lower bounded by a positive constant. Consequently, reliable cooperation cannot be achieved, as the relay does not transmit any information to the destination when it fails to decode the received signal, and the destination cannot reliably decode the signal received from the source, since $\sigma_{\mathrm{SD}}>\sigma_{\mathrm{SR}}>\sigma_{1}^{*}(\lambda, \rho)$.

From now on we consider that $\sigma_{\mathrm{SR}}<\sigma_{1}^{*}(\lambda, \rho)$; hence, we may assume error free 4 transmission between source and relay. We will also use the following notation:

- $\sigma=\sigma_{\mathrm{RD}}$ (we drop subscript $\mathrm{RD}$ ), which will be referred to as noise parameter,

- $\delta=\frac{\sigma_{\mathrm{SD}}}{\sigma_{\mathrm{RD}}} \geq 1$, which will be referred to as channel discrepancy.

The threshold function $\sigma_{\theta, \lambda, \rho}^{*}:[1, \infty[\rightarrow \mathbb{R}$ associates with each discrepancy value $\delta$ the noise threshold $\sigma_{\theta, \lambda, \rho}^{*}(\delta)$, defined as the supremum value of $\sigma$ that allows transmission with an arbitrary small error probability, assuming that the transmitted data is encoded with an arbitrary-length distributed code from $\mathcal{E}_{\theta}(\lambda, \rho)$. This definition makes sense only under the implicit assumption of a concentration theorem as in [16], which can indeed be derived by using the same arguments as in loc. cit.

The threshold function can be efficiently computed by tracking the density evolution of messages exchanged within the iterative decoding, as explained in the next section.

\section{B. Density evolution}

Throughout this section, we assume binary-input AWGN relay channel. We combine a multi-edge approach [17] and the Gaussian approximation method proposed in [18], in order to derive density evolution equations for the SE-LDPC code ensemble $\mathcal{E}_{\theta}(\lambda, \rho)$. We separate the set of bit-nodes of the expanded graph into two subsets:

- type-1 bit-nodes, which correspond to bits received by the destination from the source,

- type-2 bit-nodes, which correspond to extended bits received by the destination from the relay.

We distinguish between type- 1 and type- 2 edges, according to whether they are incident to type- 1 or type- 2 bit-nodes. Moreover, check-node degrees are also defined type-wise. Hence, we say that a check-node is of degree $\left(d_{1}, d_{2}\right)$ if it is connected to $d_{1}$ type- 1 bit-nodes and $d_{2}$ type- 2 bit-nodes. From our definition of SE-LDPC ensembles, it follows that the type- 2 degree $d_{2}$ is equal either to 1 or 2 (see Fig. 2). Finally, for each type $t=1,2$, we define:

- $\lambda_{d}^{[t]}$ is the fraction of type- $t$ edges connected to bit-nodes of degree $d$,

- $\rho_{d_{1}, d_{2}}^{[t]}$ is the fraction of type- $t$ edges connected to checknodes of degree $\left(d_{1}, d_{2}\right)$.

\footnotetext{
${ }^{4}$ With arbitrarily small error probability, as the code length tends to infinity.
} 
It follows that $\lambda_{d}^{[1]}=\lambda_{d}, \lambda_{2}^{[2]}=1\left(\lambda_{d}^{[2]}=0\right.$ for $\left.d \neq 2\right)$, while $\rho_{d, i}^{[1]}$ and $\rho_{d, i}^{[2]}(i=1,2)$ can be computed as follows:

$$
\begin{array}{ll}
\rho_{d, 1}^{[1]}=d \sum_{j=-\theta+1}^{\theta-1} k_{j, 1} \frac{\rho_{d \theta+j}}{d \theta+j}, & \rho_{d, 1}^{[2]}=\frac{\bar{\rho} \rho_{d, 1}^{[1]}}{2 d(\theta-1)}, \\
\rho_{d, 2}^{[1]}=d \sum_{j=-\theta+1}^{\theta-1} k_{j, 2} \frac{\rho_{d \theta+j}}{d \theta+j}, & \rho_{d, 2}^{[2]}=\frac{\bar{\rho} \rho_{d, 2}^{[1]}}{d(\theta-1)},
\end{array}
$$

where $\bar{\rho}=\frac{1}{\int_{0}^{1} \rho(x) \mathrm{d} x}$ is the average check-node degree of the original (unsplit) graph, and

$$
\begin{aligned}
& k_{j, 1}=\left\{\begin{array}{cl}
0, & \text { if } 2 \leq j \leq \theta-1 \\
1, & \text { if } j=1 \text { or } j=-\theta+1 \\
2, & \text { if }-\theta+1<j \leq 0
\end{array}\right. \\
& k_{j, 2}=\theta-|j|-k_{j, 1}
\end{aligned}
$$

Now, under the Belief-Propagation decoding, let $m_{v^{[t]}}^{(\ell)}$ denote the mean of outgoing messages from type- $t$ bit-nodes at iteration $\ell$. Let also $r_{\ell}^{[t]}=1-E\left(\tanh \frac{m_{v[t]}^{(\ell)}}{2}\right)$, where $E$ denotes as usual the expected value operator. Define:

$$
\begin{aligned}
\phi(x) & =1-\frac{1}{2 \sqrt{(\pi x)}} \int_{\mathbb{R}} \tanh \frac{u}{2} e^{-\frac{(u-x)^{2}}{4 x}} \mathrm{~d} u,(\phi(0)=1) \\
\psi(x) & =\phi^{-1}(1-x) \\
h^{[1]}(x, y) & =\sum_{j} \lambda_{j}^{[1]} \phi\left(\frac{2}{(\delta \sigma)^{2}}+(j-1) \sum_{i_{1}, i_{2}} \rho_{i_{1}, i_{2}}^{[1]} \psi\left(x^{i_{1}-1} y^{i_{2}}\right)\right) \\
h^{[2]}(x, y) & =\sum_{j} \lambda_{j}^{[2]} \phi\left(\frac{2}{\sigma^{2}}+(j-1) \sum_{i_{1}, i_{2}} \rho_{i_{1}, i_{2}}^{[2]} \psi\left(x^{i_{1}} y^{i_{2}-1}\right)\right)
\end{aligned}
$$

Then, under the assumption that the messages exchanged during the iterative Belief-Propagation decoding are independent and symmetric Gaussian distributed, $r_{\ell}^{[1]}$ and $r_{\ell}^{[2]}$ can be recursively computed by:

$$
\left(r_{\ell}^{[1]}, r_{\ell}^{[2]}\right)=\left(h^{[1]}\left(1-r_{\ell-1}^{[1]}, 1-r_{\ell-1}^{[2]}\right), \quad h^{[2]}\left(1-r_{\ell-1}^{[1]}, 1-r_{\ell-1}^{[2]}\right)\right),
$$

with initial values $\left(r_{0}^{[1]}, r_{0}^{[2]}\right)=\left(\phi\left(\frac{2}{(\delta \sigma)^{2}}\right), \phi\left(\frac{2}{\sigma^{2}}\right)\right)$. The proof will be omitted, since it follows from the same arguments as in [18]. The above recursion holds as long as $\ell$ is less than half the girth of the graph, which goes to infinity with the code-length, and the successful decoding condition for an "infinite" code from $\mathcal{E}_{\theta}(\lambda, \rho)$ can be expressed as $\left(r_{\ell}^{[1]}, r_{\ell}^{[2]}\right) \rightarrow 0$. Therefore, the threshold function defined in the above section, can be computed by:

$$
\sigma_{\theta, \lambda, \rho}^{*}(\delta)=\sup \left\{\sigma \mid \lim _{\ell \rightarrow \infty} r_{\ell}^{[1]}=\lim _{\ell \rightarrow \infty} r_{\ell}^{[2]}=0\right\}
$$

\section{Theoretical limit}

In order to evaluate the performance of an ensemble of codes, we would like to compare its threshold function with the capacity function, inferred from the channel capacity.

Capacities of various relaying strategies have been computed in [19], [20], [21], and depend on the capacities of the three links. Since we assumed that source-to-relay transmission is error free 5 , we only consider the two other links. Let $\gamma_{\mathrm{RD}}(\sigma)$ denote the information rate capacity of the relay-todestination channel with parameter $\sigma$, and let $\gamma_{\mathrm{SD}}(\sigma)$ be defined in a similar manner. The information rates are considered by transmitted bit, thus both $\gamma_{\mathrm{RD}}(\sigma), \gamma_{\mathrm{SD}}(\sigma) \in[0,1]$. We also assume that the noise parameter $\sigma \in\left[0,+\infty\left[\right.\right.$ and $\gamma_{\mathrm{RD}}, \gamma_{\mathrm{SD}}$ are continuous decreasing functions, such that $\gamma_{\mathrm{RD}}(0)=\gamma_{\mathrm{SD}}(0)=$ 1 and $\lim _{\sigma \rightarrow+\infty} \gamma_{\mathrm{RD}}(\sigma)=\lim _{\sigma \rightarrow+\infty} \gamma_{\mathrm{SD}}(\sigma)=0$.

Now, let $\left(r_{1}, r_{2}\right) \in[0,1] \times[0,+\infty[$ be a target distributed coding rate. The capacity function $\gamma_{r_{1}, r_{2}}:[1,+\infty[\rightarrow[0,+\infty[$ is defined by $\gamma_{r_{1}, r_{2}}(\delta)=\sigma$, where $\sigma$ is the unique solution of the equation:

$$
\frac{\gamma_{\mathrm{SD}}(\delta \sigma)}{r_{1}}+\frac{\gamma_{\mathrm{RD}}(\sigma)}{r_{2}}=1
$$

Note that for $\sigma=0$, we have $\frac{\gamma_{\mathrm{SD}}(0)}{r_{1}}+\frac{\gamma_{\mathrm{RD}}(0)}{r_{2}}=\frac{1}{r_{1}}+\frac{1}{r_{2}} \geq$ $\frac{1}{r_{1}} \geq 1$, and $\lim _{\sigma \rightarrow+\infty} \frac{\gamma_{\mathrm{SD}}(\delta \sigma)}{r_{1}}+\frac{\gamma_{\mathrm{RD}}(\sigma)}{r_{2}}=0$, thus such a solution always exists and it is unique, due to the above assumptions.

The meaning of the capacity function is the following. Assume that we want to transmit information with distributed rate $\left(r_{1}, r_{2}\right)$ over some relay channel. The rate $r_{1}$ is chosen according to the quality of the channel between source and relay, such that to ensure error free transmission between them. The rate $r_{2}$ is generally chosen according to the delay constraints of the cooperation system. The question is to know is there exists a distributed code with distributed rate $\left(r_{1}, r_{2}\right)$ allowing error free transmission. The answer is as follows. If the discrepancy and noise parameters $(\delta, \sigma)$ of the relay channel verify $\sigma<\gamma_{r_{1}, r_{2}}(\delta)$ then such a distributed code exits. However, note that a code allowing error free transmission for some pair $(\delta, \sigma)$, might not be suitable for some other pair of parameters satisfying the above condition. If $\sigma>\gamma_{r_{1}, r_{2}}(\delta)$, then reliable transmission with distributed rate $\left(r_{1}, r_{2}\right)$ is not possible. The proof will be given in an extended version of this paper.

\section{NUMERICAL RESULTS}

We assume BI-AWGN relay channel throughout this section. The following degree distribution pair, with designed coding rate $1 / 2$, was designed by exact density evolution, and its threshold 7 over the BI-AWGN channel is $\sigma^{*}=0.9649$ [5].

$$
\begin{aligned}
\lambda(x)= & 0.2199 x+0.2333 x^{2}+0.0206 x^{3}+0.0854 x^{5}+0.0654 x^{6} \\
& +0.0477 x^{7}+0.0191 x^{8}+0.0806 x^{18}+0.2280 x^{19} \\
\rho(x)= & 0.6485 x^{7}+0.3475 x^{8}+0.0040 x^{9}
\end{aligned}
$$

First, we consider the SE-LDPC code ensemble $\mathcal{E}_{2}(\lambda, \rho)$. The designed distributed rate is $\left(r_{1}, r_{2}\right)=(1 / 2,1)$, meaning that the source broadcasts a code with rate $1 / 2$, and the relay generates a number of extended-bits equal to the number of information bits. If the standard deviation of the white Gaussian noise on the source-to-relay link is less than the

\footnotetext{
${ }^{5}$ In practice, the channel need not be error free; the assumption is that the $\sigma_{\mathrm{SR}}$ noise is below the threshold of the code broadcasted by the source.

${ }^{6}$ Arbitrary small error probability when the code length goes to infinity.

${ }^{7}$ Note that the threshold calculated by Gaussian approximation is 0.9459 .
} 
above threshold $\sigma^{*}$ or, equivalently, the signal to noise ratio is greater than $\mathrm{SNR}^{*}=-2.70 \mathrm{~dB} 8$, we can assume error free transmission between source and relay. From our definition of the discrepancy, the signal to noise ratios on the sourceto-destination and relay-to-destination links, are related by $\mathrm{SNR}_{\mathrm{RD}}=\mathrm{SNR}_{\mathrm{SD}}+\Delta$, where $\Delta=10 \log _{10}\left(\delta^{2}\right)$ is the discrepancy value in $\mathrm{dB}$. The ensemble threshold and the capacity functions are plotted at Figure 6 We can observe that the gap between the two curves is relatively small (between 0.7 and $0.3 \mathrm{~dB}$ ) for discrepancy values $\Delta \in[0,8.5]$, and it begins to increase starting from this point. Hence, if a discrepancy value $\Delta>8.5 \mathrm{~dB}$ is not likely to be encountered in practice, the above SE-LDPC code can be used to achieve reliable communication for channel parameters $(\delta, \sigma)$ very close to the capacity.

[Remark] HARQ systems with incremental redundancy represent another possible application of the proposed SE-LDPC codes. In this case extended-bits are transmitted by the source as incremental redundancy, whenever the destination fails to decode the originally received signal. In such a case, the discrepancy is expected to take on relatively small values.

Figure 6 shows also the threshold function for the SELDPC code ensemble $\mathcal{E}_{3}(\lambda, \rho)$, whose designed distributed rate is $\left(r_{1}, r_{2}\right)=(1 / 2,1 / 2)$. We can observe that the gap between the threshold and the capacity curves is between 1 and $0.4 \mathrm{~dB}$ for discrepancy values $\Delta \in[0,20]$. This proves that split-extending good codes for point-to-point communications results in good distributed codes for cooperative communications.

\section{Conclusions}

We proposed a new code-design method for LDPC coded cooperation, which is based on a split-and-extend approach. First, we showed that the proposed design can be advantageously applied to existing codes, which allows for addressing cooperation issues for evolving standards. Subsequently, we introduced the concepts of threshold and capacity functions, and we derived density evolution equations for split-extended codes. Some ensemble thresholds have been presented, showing that codes optimized for point-to-point communications can be split-extended, so that the corresponding distributed codes perform close to the capacity of the relay channel for a wide range of discrepancy values. Optimization of splitextended codes will be addressed in future works. Finally, besides advantageous applications for cooperative transmission systems, the proposed design can also be used for communication systems employing HARQ schemes with incremental redundancy.

\section{REFERENCES}

[1] A. Sendonaris, E. Erkip, and B. Aazhang, "User cooperation diversity. Part I. System description," IEEE Transactions on Communications, vol. 51, no. 11, pp. 1927-1938, 2003.

[2] A. Sendonaris, E. Erkip, and B. Aazhang, "User cooperation diversity. Part II. Implementation aspects and performance analysis," IEEE Transactions on Communications, vol. 51, no. 11, pp. 1939-1948, 2003.

\footnotetext{
${ }^{8}$ Note that the theoretical limit is at $-2.82 \mathrm{~dB}$.
}

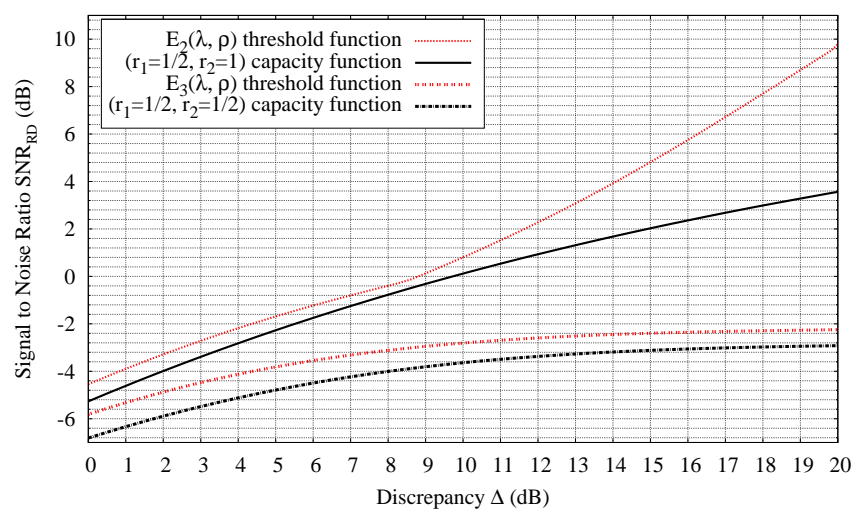

Fig. 6. Threshold vs. capacity function

[3] J.N. Laneman, D.N. Tse, and G.W. Wornell, "Cooperative diversity in wireless networks: efficient protocols and outage behaviour," IEEE Trans. on Information Theory, vol. 50, no. 12, pp. 3062-3080, 2004.

[4] M. C. Valenti and B. Zhao, "Distributed turbo codes: towards the capacity of the relay channel," in IEEE Vehicular Technology Conference (VTC), 2003, pp. 322-326.

[5] T. J. Richardson, M. A. Shokrollahi, and R. L. Urbanke, "Design of capacity approaching irregular low density parity check codes," IEEE Trans. Inform. Theory, vol. 47, no. 2, pp. 619-637, 2001.

[6] M. A. Khojastepour, N. Ahmed, and B. Aazhang, "Code design for the relay channel and factor graph decoding," in Asilomar Conf. on Signals, Systems and Computers, 2004, pp. 2000-2004.

[7] P. Razaghi and W. Yu, "Bilayer LDPC codes for the relay channel," in IEEE Inter. Conf. on Communications (ICC), 2006, pp. 1574-1579.

[8] P. Razaghi and W. Yu, "Bilayer low-density parity-check codes for decode-and-forward in relay channels," IEEE Trans. on Information Theory, vol. 53, no. 10, pp. 3723-3739, 2007.

[9] A. Chakrabarti, A. De Baynast, A. Sabharwal, and B. Aazhang, "Lowdensity parity-check codes for the relay channels," IEEE Journal on Selected Areas in Communications, vol. 25, no. 2, pp. 280-291, 2007.

[10] J. Hu and T. M. Duman, "Low density parity check codes over wireless relay channels," IEEE Trans. on Wireless Communications, vol. 6, no. 9, pp. 3384-3394, 2007.

[11] C. Li, G. Yue, M. A. Khojastepour, X. Wang, and M. Madihian, "LDPC coded cooperative relay systems: performance analysis and code design," IEEE Trans. on Communications, vol. 56, no. 3, pp. 485-496, 2008.

[12] J. Cances and V. Meghdadi, "Optimized low density parity check codes designs for half duplex relay channels," IEEE Trans. on Wireless Communications, vol. 8, no. 7, pp. 3390-3395, 2009.

[13] D. Duyck, J. J. Boutros, and M. Moeneclaey, "Low-density paritycheck coding for block fading relay channels," in IEEE Inform. Theory Workshop (ITW), 2009, pp. 248-252.

[14] IEEE-802.16e, "Physical and medium access control layers for combined fixed and mobile operation in licensed bands," 2005, amendment to Air Interface for Fixed Broadband Wireless Access Systems.

[15] V. Savin, "Self-corrected min-sum decoding of LDPC codes," in IEEE Int. Symp. on Inform. Theory (ISIT), 2008, pp. 146-150.

[16] T. J. Richardson and R. L. Urbanke, "The capacity of low-density paritycheck codes under message-passing decoding," IEEE Trans. Inform. Theory, vol. 47, no. 2, pp. 599-618, 2001.

[17] T. J. Richardson and R. L. Urbanke, "Multi-edge type LDPC codes," Available online: http://lthcwww.epfl.ch/papers/multiedge.ps.

[18] S.-Y. Chung, T.J. Richardson, and R.L. Urbanke, "Analysis of sumproduct decoding of low-density parity-check codes using a Gaussian approximation," IEEE Trans. Inform. Theory, vol. 47, no. 2, pp. 657670, 2001

[19] T. Cover and A. E. Gamal, "Capacity theorems for the relay channel," IEEE Trans. on Information Theory, vol. 25, no. 5, pp. 572-584, 1979.

[20] M. A. Khojastepour, A. Sabharwal, and B. Aazhang, "On capacity of Gaussian 'cheap' relay channel," in IEEE Global Telecom. Conference (GLOBCOM), 2003, vol. 3, pp. 1776-1780.

[21] G. Kramer, M. Gastpar, and P. Gupta, "Cooperative strategies and capacity theorems for relay networks," IEEE Trans. on Information Theory, vol. 51, no. 9, pp. 3037-3063, 2005. 